Colloque international « 1945, le retour des films américains en Europe : économie, politique, esthétique »

Université de Lausanne, 7-8 mars 2013

\title{
Charlotte Rey
}

\section{(2) OpenEdition}

\section{Journals}

\section{Édition électronique}

URL : https://journals.openedition.org/1895/4688

DOI : $10.4000 / 1895.4688$

ISSN : 1960-6176

\section{Éditeur}

Association française de recherche sur l'histoire du cinéma (AFRHC)

\section{Édition imprimée}

Date de publication : 1 juin 2013

Pagination : 175-179

ISBN : 978-2-37029-070-0

ISSN : 0769-0959

\section{Référence électronique}

Charlotte Rey, «Colloque international « 1945, le retour des films américains en Europe : économie, politique, esthétique » », 1895. Mille huit cent quatre-vingt-quinze [En ligne], 70 | 2013, mis en ligne le 01 juin 2016, consulté le 15 avril 2022. URL : http://journals.openedition.org/1895/4688 ; DOI : https:// doi.org/10.4000/1895.4688 
issus de la cybernétique. Krichane a ainsi éclairé d'une perspective nouvelle la notion de "code» afin de proposer, à partir de Metz, une sémiologie des jeux vidéos. Cette volonté de revitaliser et d'appliquer la théorie metzienne a également été au centre de la présentation de Roger Odin qui, en ouverture du colloque, appelait de ses vœux un renouveau de l'axe de recherche sur le langage cinématographique à la lumière des pratiques filmiques actuelles.

Que reste-t-il de Metz aujourd'hui? En sourdine tout au long du colloque, cette question trouve en définitive sa réponse au croisement des multiples interventions. Se pose-t-elle différemment aujourd'hui qu'en 1989 lors d'un premier colloque Metz à Cerisy? Sans doute dans la mesure où l'importance singulière de ses archives dévoile une "réserve» de textes insoupçonnée des disciples et des commentateurs. Dès lors l'œuvre de Metz se profile peut-être aujourd'hui davantage comme un objet historique que comme un modèle théorique. D'autant plus que ces inédits vont susciter une infinité de commentaires propres à redessiner les contours de l'œuvre... Dans la pléthore de ces fiches personnelles, notes et variantes, on devine un objet complexe: le modèle théorique de Metz sous l'éclairage de Christian.

Charlotte Bouchez, Omar Hachemi
Colloque international «1945, le retour des films américains en Europe: économie, politique, esthétique» (Université de Lausanne, 7-8 mars 2013)

LES 7 ET 8 MARS DERNIERS, s'est tenu à l'Université de Lausanne le colloque international «1945, le retour des films américains en Europe: économie, politique, esthétique» codirigé par François Albera (Lausanne), Carine Bernasconi (Lausanne) et Laurent Le Forestier (Rennes 2). Ces deux jours ont permis de faire le point sur les relations entre différents pays européens et Hollywood au sortir de la Seconde Guerre mondiale. Comme l'indiquait le sous-titre du colloque, les principaux axes développés ont été des questions politiques et économiques, les interrogations d'ordre esthétique, quant à elles, ont été prises en considération lorsqu'elles étaient clairement déterminées par le contexte socio-politique. L'apport majeur de cette rencontre a été la mise au jour des liens entre l'industrie, la politique et la diffusion de cinémas nationaux dans différents pays européens (Belgique, Danemark, France, Espagne, Italie, Suisse et Tchécoslovaquie) dans les années 1940 et 1950. Les points communs ainsi que les divergences des stratégies américaines concernant les politiques d'exploitation et de diffusion des films hollywoodiens ont également été au centre des débats. La réception des films européens dans ces contextes de cohabitation avec les Majors et les différents offices étatiques américains (MPAA, MPEA, PWB) ont aussi fait l'objet d'analyses qui ont relevé toute la complexité des rapports entre le public, les instances étatiques nationales et américaines, ainsi qu'avec l'industrie cinématographique elle-même.

Pour compléter ces deux journées, une soirée de projections a été organisée en collaboration avec la Cinémathèque suisse, durant laquelle a été présenté The Search (les Anges marqués) de Fred Zinnemann (1948), un film produit par la Praesens de Zürich dont le rôle est essentiel dans le cinéma suisse des années 1940 et 1950. Co-production helvéticoaméricaine qui met en scène un soldat américain en Allemagne en 1946 (Montgomery Clift), ce film fait partie des productions dont les revenus ont été 
réinvestis par les États-Unis dans le pays où des capitaux avaient été engagés auparavant. En avantprogramme était projeté Grande semaine du film américain à Lausanne (Ciné-Journal suisse, 1946) et Deutschland heute, Schweizer Spende. Reportage en Allemagne (Ciné-Journal suisse, 21 octobre 1947). Le second reportage comprenait un film de promotion de The Search financé par le Don suisse (Schweitzer Spende) promouvant non seulement le film mais également le pays et son industrie cinématographique. Le Diable au corps (1946) de Claude Autant-Lara, dont une partie du financement est également états-unien (mais de façon plus indirecte que le film de Zinnemann) a été projeté en deuxième partie de soirée, précédé d'Images du cinéma français (Ciné-Journal suisse, septembre 1945).

Lintroduction des trois co-organisateurs a permis de pointer des éléments historiographiques et contextuels pour le cas français d'abord, qui a été envisagé comme point de départ de ces rencontres; elle a également brossé le contexte historiographique européen dans un second temps. Ces présentations sur le retour des films américains en France ont permis de rappeler la richesse des sources encore à explorer et la complexité historiographique du statut de cette période dans l'Histoire du cinéma. Le panorama des études existantes en Europe a présenté l'engouement pour ces questions dans les années 1990, en écho aux accords du GATT conclus à cette période. Entre 1987 et 1993, un certain nombre de conférences ont été organisées en Angleterre, en Italie et aux États-Unis. Toutes cherchent à réévaluer le discours dominant tenant à placer Hollywood dans un rapport purement mercantile et manichéen vis-àvis du Vieux Continent, et il se dessine un déplacement des questionnements sur le sujet. Le constat face aux politiques américaines de l'époque apparaît nettement plus nuancé qu'il n'était dépeint au premier abord.

Durant la première journée, la plupart des communications ont porté sur des questions liées à la France. La confrontation des exposés de Guillaume Vernet (Rennes 2) et François Garçon (Paris 1) a soulevé et renouvelé la complexité de l'analyse de la réception des films américains en France, ainsi que la position de l'industrie face à la politique de diffusion de ces films sur le territoire national. En outre, les relations entretenues par les membres de l'industrie cinématographique française, notamment par ceux rattachés ou proches du PCF, restent un enjeu essentiel dans la compréhension de la période, et l'analyse de ces relations diverge en fonction des sources considérées. Vernet au travers de sa communication : "La "défense du cinéma français" par "ceux qui le font”. Mobilisation de la profession pour la révision des accords Blum-Byrnes et construction d'un modèle cinématographique national (19471948 ) ", a proposé de discuter de la reconstruction du cinéma français avec comme point de départ les accords franco-américains, en reconsidérant l'idée de «colonisation culturelle» de la part d'Hollywood dans la France d'après-guerre. De son analyse, essentiellement fondée sur des discours de membres de l'industrie cinématographique, découle l'idée d'une reconstruction du cinéma français à partir du cinéma américain plutôt qu'en réaction à lui. Garçon traitant de «L'éternel retour des communistes dans le cinéma français: les accords Blum-Byrnes", a proposé de réévaluer les positions et les discours anti-américains de toute une partie de la profession liée au PCF, souvent décrits dans les histoires sur cette période comme dominants. Il a été plus largement présenté une interrogation sur la manière de percevoir les accords Blum-Byrnes en nuançant certaines idées transmises par la presse de l'époque. Les sources prises en compte dans cette communication s'écartaient de celle choisies par Vernet dans la mesure où il ne s'agissait pas de discours issus des milieux professionnels français, mais d'éléments d'archives administratives.

Au travers de «La programmation des ciné-clubs d'après-guerre ou la constitution d'un panthéon cinéclubiste à l'ombre du cinéma hollywoodien", sujet auquel il s'intéresse dans le cadre de sa thèse, Léo Souillés (Université de Lorraine) a présenté les tensions existantes entre les ciné-clubs et leurs publics, oscillant entre une volonté de «bon cinéma» et la découverte d'un cinéma impossible à voir sous 
l'Occupation, mais qui ferait courir à sa perte l'industrie nationale. Basée sur l'étude statistique des programmations de ciné-club et d'analyses de discours, son analyse a mis au jour les frictions entre rejet et fascination pour Hollywood qui sont apparues sur un autre plan.

Par ailleurs, les exposés de Roxane Hamery (Rennes 2), "Le film criminel américain, école de gangstérisme? Débats sur la jeunesse délinquante et le cinéma» et Gaétan Raoul (Rennes 2), "L'influence du modèle américain dans les films français du plan Marshall consacrés à l'agriculture», se sont penchés sur des questions périphériques, permettant d'aborder des questions non plus directement liées au débat sur la place des films américains dans l'après-guerre en France, mais sur les applications - symptômes et tensions - qui résident dans l'influence des États-Unis sur le Vieux Continent. Par le croisement d'études de criminologies, de pédagogies et de critiques cinématographiques, Hamery a présenté la manière dont étaient perçus le film de gangster américain ainsi que le film policier français. Dangereux pour la jeunesse, car promouvant un modèle ambigu de moralité, ces œuvres se voient imputer la hausse de la délinquance. Cette conséquence sociale apparaît de manière générale dans la société française, le film hollywoodien étant pointé du doigt dans sa volonté supposée de chercher à faire valoir un nouveau mode de vie et de moralité. Raoul s'est quant à lui penché sur l'influence, aussi bien esthétique que thématique, du modèle américain dans les films agricoles français, des productions directement issues des directives du plan Marshall.

Les deux autres communications de cette première journée ont été conduites par Fernando González (Salamanque) et Jindriska Blahova (Université Masaryk, Brno). Celle portant sur «La difficile adoption des formes américaines par le cinéma espagnol des années 40 » s'est intéressée à l'esthétique du cinéma espagnol dès 1942 en relation avec le modèle hollywoodien et les questions de censure formelles de l'époque. En partant d'un constat de l'état de la production et de la diffusion en Espagne dans l'après-guerre cette communication a été l'occasion de mettre en avant les résistances du cinéma nationale à se conformer aux canons hollywoodiens. Selon González, le cinéma espagnol, financé dès ces années par les films américains doublés, garde une mise en scène et un montage issus de la tradition scénique populaire. La forme "théâtralisée» des films de ces années découle également d'une censure formelle imposée par les commissions d'évaluation de censure et d'un goût du public pour le texte. La communication de Blahova intitulée «A Tough Job for Donald Duck: Selling Films behind the Iron Curtain, 1944-1951", a montré l'importance des relations qu'entretenait le gouvernement américain, principalement en la personne d'Eric Johnston - président du MPAA -, avec le bloc soviétique. Dès la fin de la Seconde Guerre mondiale, se met en place à Hollywood le «Soviet Sphere Project», conduit par le Motion Picture Export Association (MPEA), présent dans de nombreux pays européens. La présence d'une communication constante entre les États-Unis, profondément anti-communistes, avec les antennes de Moscou, montre, au travers de sources administratives américaines, le projet de globalisation désiré par Hollywood de mettre à mal l'industrie cinématographique communiste et d'utiliser le cinéma comme arme de propagande.

La deuxième journée du colloque a été ouverte par la communication de Pierre-Emmanuel Jaques (Lausanne) «Entre dithyrambe et anathème: la réception du cinéma américain dans la presse suisse romande (1945-1948)», traitant du cas suisse. Celleci a permis de faire le lien d'une part avec des questions posées au travers du cas français - la visibilité des films hollywoodiens durant la guerre en Suisse était relayée dans une presse lue en France et construisait l'imaginaire du star system dans le pays occupé -, et d'autre part avec les projections ayant eu lieu à la Cinémathèque suisse la veille. Après un bref panorama du contexte de la production et de la diffusion cinématographique en Suisse durant la guerre, considérée souvent comme l'Âge d'or du cinéma helvétique, l'analyse de la réception de l'après-guerre a montré la place faite au cinéma étranger. La visibilité du cinéma international n'est pas complètement 
exempte de censure, certains critiques appartenaient d'ailleurs à des commissions cantonales, tout en se donnant pour mission de guider le spectateur, plutôt que de juger ou louer. Si, avant 1945, le cinéma américain reçoit une presse plutôt positive, ce point de vue n'est plus si unanime dans la seconde moitié des années 1940. Les productions étrangères interdites sont le plus souvent américaines, mais aussi françaises (Zéro de conduite, Un chien andalou), ou italienne (Ossessione - les Amants diaboliques). Il apparaît également une confrontation entre le cinéma français et le cinéma américain, le premier étant perçu comme pessimiste, amoral et posant des questions sociales, alors que le second est décrit comme optimiste et moral. D’ailleurs au travers du néo-réalisme, la question du véridique est récurrente durant cette période. Les positions critiques divergentes en fonction des positionnements politiques reprennent également cette thématique discursive.

Ont suivi deux exposés sur le cas italien. La présentation de Francesca Leonardi (Paris 3), "Les films hollywoodiens débarquent en Italie: modalités de diffusion et réception", s'est attachée à analyser des sources provenant du Psychological Warfare Branch ainsi que de la presse italienne et américaine, afin de discuter la collaboration délicate entre les Majors, l'OWI (Office of War Information) et l'armée à propos de la diffusion des films sur le territoire italien. Ces modes de diffusion, définis dans une intention propagandiste, ont été comparés à la réception dans la presse. Leonardi ayant dépeint le contexte de diffusion et un état de la réception des films entre juillet 1943 et octobre 1945, Paola Palma (Vérone) s'est intéressée à la question de la censure dans les films américains sortis en Italie entre 1943 et 1952. Les commissions de censure sont d'une part du côté des organismes américains qui refusent les films valorisant le fascisme, d'autre part, elles restent italiennes, notamment au travers du CCC (le Centre catholique cinématographique). Dès 1934 déjà, l'interdiction des films en langue étrangère permet des modifications importantes du sens des films américains. Se développe alors une censure par la coupe, mais également par le doublage. Ces diffé- rentes manipulations ou "soustractions» - changements de nationalités ou disparitions des personnages italiens négatifs; nuances ou élimination des critiques touchant au clergé, ou des discours défendant les droits et les valeurs populaires; mais aussi atténuation de l'érotisme; discours prônant l'entrée en guerre ou même les valeurs démocratiques sont des éléments ayant un impact sur la réception des films et sur la perception du cinéma américain en général. Un certains nombre d'exemples ont permis d'illustrer ce propos tel Grapes of Warth (les Raisins de la colère) de John Ford produit en 1940, sorti en Italie en 1952, dont la scène finale a disparu de la version italienne.

Enfin, les deux dernières communications de ces journées se sont arrêtées sur le Danemark et la Belgique. Chacune a présenté une analyse de la diffusion et de la production du cinéma national et étranger au retour du cinéma hollywoodien. Les deux situations ont clairement exprimé les différences majeures de stratégie du SHAEF (Supreme Headquarter Allied Expeditionary Forces) et de la politique nationale face aux changements engendrés par la reconfiguration du panorama cinématographi-que. Isak Thorsen (Copenhague) a mis en évidence les difficultés de cohabitation entre les Majors américaines et les distributeurs danois entre 1945 et 1958. En effet, durant cette période, trois blocus des productions américaines ont eu lieu sur le territoire danois, transformant régulièrement le paysage cinématographique. Le Danemark, qui sort riche et une industrie cinématographique nationale florissante de la guerre, voit sa production nationale mise à mal par le retour sur le territoire des huit Majors collaborant étroitement avec les bureaux d'État américains. Les blocus ont pour origine l'augmentation des coûts de location des films aux distributeurs nationaux et ont principalement impliqué une renégociation de l'utilisation des capitaux étrangers. Du point de vue de la diffusion, ils ont fait place au cinéma européen. Toutefois, le désir du public de voir les productions américaines a créé un commerce maritime entre le pays et la Suède, appelé phénomène des Scarlett-Lines. En outre, la compagnie du même nom coordonnait 
ses traversées avec les projections de films américains diffusés sur le sol suédois.

Au contraire du Royaume du Danemark, dont la configuration du paysage cinématographique a surtout été dictée par des raisons économiques, la Belgique est soumise à des pressions censoriales de l'État (il s'agit de contrôle plus que de censure) et par des commissions catholiques peu restrictives. L'exposé de Daniel Biltereyst a montré que l'intérêt du marché belge pour l'industrie américaine était dû à la forte densité de salles, sans qu'existe pour autant une industrie nationale. Sa communication s'est concentrée sur la réception historique, l'import et la diffusion des films américains sur le territoire belge entre septembre 1944 et 1955 . Les sources utilisées pour l'analyse des données sont d'une part les archives de presse, mais également des données provenant d'un projet de recherche portant sur le bureau national de censure du film belge, ayant pour objectif de comprendre précisément les raisons de censure (autorisation des films pour un public adulte et/ou coupe) des productions hollywoodiennes, mais aussi européennes. Il ressort de cette analyse une préférence pour le cinéma américain de la part des instances catholiques, alors qu'au niveau du contrôle étatique la moitié des productions sont considérées comme convenant à tous. Toutefois, un tiers des films doit subir des coupes pour pouvoir être montré à tous et un autre tiers reste des productions réservées à un public adulte. Par ailleurs, l'analyse de la diffusion des films dans la région de Gand a mis en avant les différents lieux de diffusion des films français et américains en corrélation avec les publics avoisinant les cinémas, permettant de préciser les types de public et relativiser la suprématie supposée d'Hollywood.

L'ensemble des communications a suscité de vifs débats et a permis d'approfondir et de réévaluer le positionnement des États-Unis vis-à-vis des marchés cinématographiques européens. Il est apparu notamment que si les offices étatiques (MPEA, MPAA, BWP et SHAEF) étaient présents sur l'ensemble du Vieux Continent, les stratégies d'assise d'Hollywood, et de ce fait d'une vision du monde capitaliste, divergeaient en fonction du pays intéressé.
La base du fonctionnement de la diffusion cinématographique américaine était commune, mais évoluait en fonction des marchés cinématographiques et des politiques de chaque pays impliqué. Les différentes approches proposées par les intervenants ont également mis en évidence la richesse de la recherche sur ces questions et le besoin de mise en commun des situations nationales, afin de construire une image européenne du retour du cinéma américain dans l'après Seconde Guerre mondiale. Des projets à l'échelle européenne permettraient d'affiner l'évaluation faite sur la place d'Hollywood dans la volonté états-unienne d'imposer son influence sur la société européenne.

Charlotte Rey 